\title{
Nuevo modelo matemático para la distribución de carga en el horno alto
}

\author{
J. Jiménez $^{(*)}$, B. Fernández ${ }^{(* *)}$, J. Sáinz de Ayala ${ }^{(* *)}$, J. Mochón ${ }^{(*)}$, A. Formoso ${ }^{(*)}$ \\ y F. Bueno ${ }^{(*)}$
}

Resumen El modelo matemático desarrollado describe la distribución de carga en el tragante de un horno alto. Se ha empleado un conjunto de variables operacionales relacionadas con el sistema de carga (Paul Wurth), junto con otras obtenidas de la carga, tales como el peso descargado, los ángulos de reposo de los materiales y el efecto del gas al atravesar el horno. Estos datos se procesan mediante herramientas informáticas. Como resultado, se obtiene la distribución de carga en el horno. Cada vez que se introduce una carga, el modelo actualiza la distribución. La aproximación empleada considera el perfil de cada capa de carga, el cual está descrito por un conjunto de polinomios interrelacionados. Los resultados se comparan con la distribución obtenida en un modelo físico del horno (escala 1/10). Tras su instalación en los ordenadores de proceso del horno alto B de Aceralia, el modelo se emplea actualmente para el control del mismo.

Palabras clave; Siderurgia. Horno alto. Control de procesos. Simulación de procesos. Modelos matemáticos. Distribución de la carga.

\section{New mathematical model for blast furnace burden distribution}

\begin{abstract}
A mathematical model for burden distribution in the blast furnace, has been developed. A set of operational variables related with the charging system (Paul Wurth) plus other parameters related with the burden itself, such as ore and coke repose angles, weights discharged and the effect of the uprising gas, have been taken into account. These data are processed by modern computer tools. As a result, the burden distribution inside the blast furnace is obtained. Every time that a charge is added to the blast furnace, the model updates the burden distribution. The approach employed is based on considering each burden layer described by a set of interrelated polynomials. It has been tested comparing its results with burden layers obtained in a physical model of the blast furnace (scale 1/10). After installation in Aceralia blast furnace B control computers, the model is currently being employed as a tool for process control.
\end{abstract}

Keywords: Ironmaking. Blast furnace. Process control. Process simulation. Mathematical models. Burden distribution.

\section{INTRODUCCIÓN}

El horno alto constituye, desde el punto de vista del problema del control de procesos, un campo fascinante, debido a la gran complejidad del proceso en sí mismo considerado. La no linealidad junto con los grandes retardos debidos a la inercia térmica y el alto nivel de ruido en las señales, propio del medio industrial, hacen que el horno alto sea difícil de controlar. Por ello, se han propuesto, a lo largo

\footnotetext{
* Centro Nacional de Investigaciones Metalúrgicas, CENIM (CSIC), Avda, de Gregorio del Amo, 8. 28040-Madrid (España).

** ACERALIA. Aptdo. 90, Gijón (Asturias, España).
}

de los últimos veinte años, modelos cuyo objeto de estudio está relacionado con uno o varios de los procesos que tienen lugar en él.

Uno de los procesos cuyo estudio más interés ha despertado es el de la distribución y movimiento de la carga en la parte superior del horno alto. Las razones son múltiples, pero cabe destacar como fundamental que la distribución de la carga en la parte superior del tragante va a determinar el comportamiento dinámico del gas a lo largo de todo el horno, comportamiento que es básico en tanto en cuanto está directamente ligado con la reducción de los minerales de hierro, así como con el estado térmico del horno. A su vez, el gas influye en la 
distribución de la carga, ya que la velocidad de salida de los gases altera el ángulo de reposo de las capas y, por tanto, la forma de éstas. Puede decirse, por consiguiente, que ambos fenómenos están fuertemente correlacionados y que su influencia es mutua. Se admite de forma generalizada que un buen control de la distribución de la carga del horno es la clave para conseguir un funcionamiento estable del mismo (1).

La importancia del control de la carga se vuelve crítica cuando se trata de hornos altos en los que se emplea como combustible adicional la inyección de carbón pulverizado por las toberas. Este proceso lleva consigo la reducción de la cantidad de coque introducido en el horno, lo que supone, a su vez, una disminución de la permeabilidad global del horno con el consiguiente riesgo de la pérdida de la estabilidad del mismo. En la actualidad, la inyección de carbón se sitúa en tasas que oscilan entre los 150 y los $180 \mathrm{~kg} / \mathrm{t}$ de arrabio producido y se estima que su límite estaría en torno a los $250 \mathrm{~kg} / \mathrm{t}$.

En el presente trabajo se describe un modelo matemático desarrollado para el estudio y la simulación de la distribución de carga en el interior del horno. Tradicionalmente, este tipo de modelos simulan la distribución final de cada capa de carga sin tener en cuenta la contribución de cada anillo. El presente modelo analiza la distribución para cada uno de los anillos de carga generados por el sistema de carga Paul Wurth ${ }^{\circledR}$. Además, permite asignar al material descargado en cada anillo un ángulo de reposo distinto, de modo que éste se adapte a las condiciones impuestas por la distribución de velocidades del gas de tragante en la dirección radial del mismo. Dicha distribución de velocidades se obtiene a partir de las sondas de análisis de gas situadas en el tragante del horno o bien a partir de los datos facilitados por un modelo específico para el análisis del flujo de gas (2).

\section{DESARROLLO DEL MODELO}

El sistema de carga para el cual se ha desarrollado el presente modelo corresponde al tragante tipo Paul Wurth. Se representa esquemáticamente en la figura 1. Básicamente consta de un sistemas de tolvas gemelas en las que se introduce el material a cargar en horno alto. Desde dichas tolvas, el material se vierte en una canaleta giratoria de ángulo de inclinación variable que distribuye la carga en el interior del horno.

Una vez que el material procedente de la canaleta entra en contacto con la superficie de carga, tenderá a acumularse formando un anillo cuyo centro coincidirá con el eje del horno. Como hipótesis de trabajo, se supone que la mayor parte del material cae en la región determinada por el punto de con-

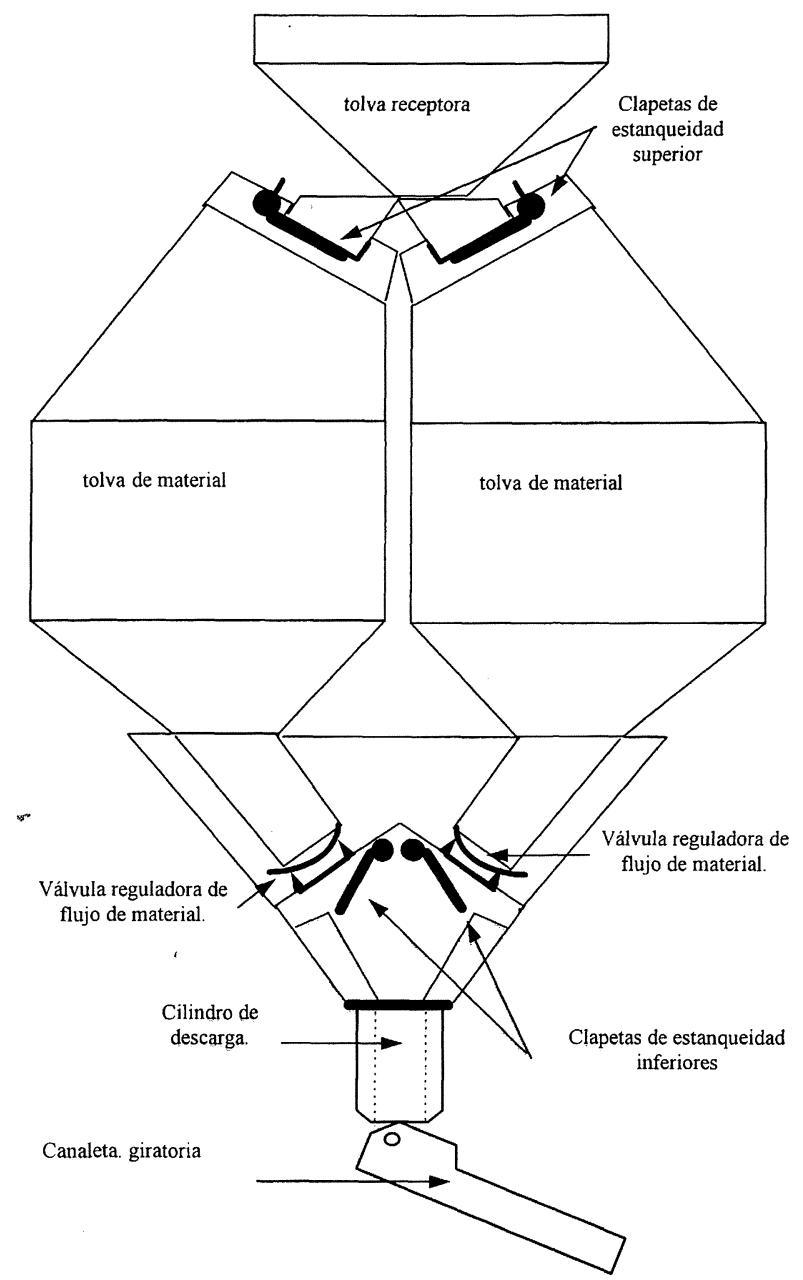

FIG. 1.- Sistema de carga tipo Paul-Wurth.

FIG. 1.-Paul-Wurth charging system.

tacto trayectoria-superficie de carga y sus proximidades. En consecuencia, el material se irá acumulando en torno a este punto, obligando a que la sección de anillo en formación vaya ganando altura paulatinamente, formando un talud exterior y otro talud interior al anillo en torno al punto de contacto. La inclinación de dicho talud vendrá determinada por el ángulo de reposo del material, Este ángulo de reposo puede definirse cualitativamente como el ángulo máximo de inclinación que se puede dar al talud formado por un determinado material sin que se derrumbe. La altura máxima y la anchura del anillo vendrán determinadas por la cantidad de material descargado. Dado que este parámetro puede variar, debido a fenómenos tales como el empuje del gas al cruzar las cargas de material, la elección de los ángulos de reposo se obtiene a partir de un modelo para el flujo de gas (2). Dicho modelo estima la velocidad superficial del gas y a partir de dicha velocidad obtiene cuál es el ángulo de reposo correcto de acuerdo con la ecuación [1] (3). 


$$
\beta=\beta_{0} \sqrt{1-\left(\frac{U}{U_{m f}}\right)^{2}}
$$

donde $\beta$ y $\beta_{0}$ representan, respectivamente, el ángulo de reposo del material con y sin paso de gas, $U$ es la velocidad del gas y $U_{\text {fm }}$, la velocidad mínima de fluidización del lecho.

Para simular la forma del perfil de cada anillo de carga, se emplea una aproximación polinómica. Con el propósito de simplificar, se eligió la aproximación polinómica de menor grado que fuera capaz de reproducir las principales características del mismo. En este caso, se construye el perfil a partir de un par de polinomios. Se asocia la posición del máximo de los mismos con la del punto de contacto entre la trayectoria del material y la de la capa anterior. Se asigna para la pendiente, en el punto de inflexión de dichos polinomios, el valor del ángulo de reposo del material, y se hace coincidir el mínimo del polinomio con el centro del horno para uno de ellos y con la pared del horno para el otro. Por último, se impone como condición que el volumen de revolución comprendido entre los polinomios así definidos y la superficie del lecho anterior coincida con el volumen del material descargado para ese anillo. El ajuste del volumen fija, a su vez, el punto de corte de los polinomios empleados con los que definen la capa anterior. En la figura 2, se muestran esquemáticamente los fundamentos del modelo que se acaba de exponer.

Se considera que la distribución de carga va a mantener la simetría cilíndrica propia del horno, de modo que el eje del mismo es de simetría para la carga.

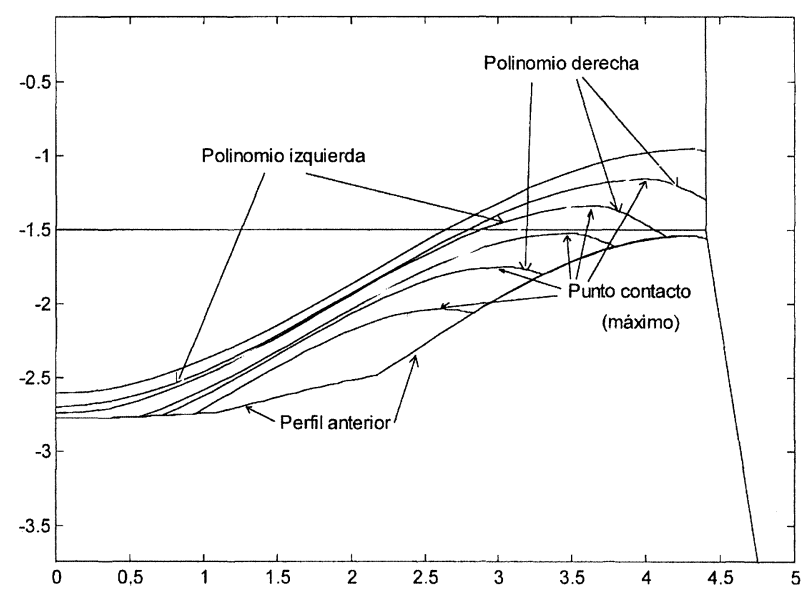

FIG. 2.- Representación esquemática del modelo empleado. La escala de los ejes corresponde a metros.

FIG. 2.- Schematic view of the model. Axis scale in meters.
Para describir la distribución de carga, y de acuerdo con la hipótesis anterior, se emplean, como ejes, el radio del horno, $r$ y la altura medida desde la línea de carga, $z$. Por razones de comodidad para los cálculos, se considera el origen de coordenadas en el centro de horno y a la altura de la línea de carga, tomando la variable $z$ valores positivos según se desciende a lo largo del horno.

Para el polinomio que describe la parte del lecho comprendida entre el centro del horno y el punto de contacto de la trayectoria con la superficie de carga se define un polinomio completo:

$$
f(r)=a_{3} r^{3}+a_{2} r^{2}+a_{1} r+a_{0}
$$

Se impone la condición de que alcance un mínimo para el centro del horno. Esto se basa en la hipótesis ya mencionada de que el centro del horno es un eje de simetría para la carga. Hay una posibilidad alternativa que se produce para el caso particular de que se esté cargando exactamente sobre la vertical del horno; en este caso, se emplea en el modelọ un polinomio del tipo de los definidos más adelante para representar el tramo de perfil comprendido entre el punto de contacto y la pared. El máximo de dicho polinomio se elegiría entonces justamente en el centro, con lo que se preservaría la simetría radial exigida para el modelo.

$$
f^{\bullet}(0)=a_{1}=0
$$

Esta primera condición de mínimo en el centro permite definir el coeficiente correspondiente al término de primer grado del polinomio igual a cero.

En segundo lugar, se exige al polinomio pasar por un máximo en el punto de contacto entre la trayectoria y la superficie de carga:

$$
f^{\prime}\left(r_{c}\right)=3 a_{3} r_{c}^{2}+2 a_{2} r_{c}+a_{1}=0
$$

$r_{\mathrm{c}}$ representa la posición de dicho punto de contacto.

Las dos condiciones siguientes se imponen sobre el punto de inflexión, que queda determinado exigiendo que la derivada segunda se anule. Además, se impone que la derivada primera en dicho punto coincida con la tangente del ángulo de inclinación del talud del material. $r_{\mathrm{i}}$ representa, por tanto, la posición del punto de inflexión.

$$
\begin{gathered}
f^{\prime}\left(r_{i}\right)=3 a_{3} r_{i}^{2}+2 a_{2} r_{i}+a_{1}=\operatorname{tg} \theta_{r} \\
f^{\prime \prime}\left(r_{i}\right)=6 a_{3} r_{i}+2 a_{2}=0
\end{gathered}
$$

Por último, se impone la condición de que el volumen comprendido entre el anillo descargado y la superficie de la carga, coincidan con el volumen de material descargado: 


$$
2 \pi \int_{r_{1}}^{r_{2}} r \cdot f(r) \cdot d r=2 \pi \int_{r_{1}}^{r_{2}} r \cdot g(r) \cdot d r
$$

donde $r_{1}$ y $r_{2}$ representan los puntos de corte entre el perfil del anillo nuevo y el perfil de carga previo y $g(r)$ representa el perfil de la carga anterior.

De las condiciones anteriores, se pueden obtener, de forma unívoca, los valores que definen los coeficientes de cada polinomio.

Efectivamente, si se resuelve el sistema de ecuaciones que se acaban de definir, se obtiene:

$$
\begin{gathered}
r_{i}=\frac{r_{0}}{2} \\
a_{3}=\frac{4 \cdot \operatorname{tg} \theta}{3 r_{0}^{2}} \\
a_{2}=\frac{2 \cdot \operatorname{tg} \theta}{r_{0}}
\end{gathered}
$$

Estos tres coeficientes definen la forma del polinomio. Queda por determinar, tan solo, el término independiente, $a_{0}$, que determina la posición relativa del polinomio definido, con respecto al eje vertical z. Se determina iterativamente hasta hacer que el polinomio definido cumpla la condición requerida de ajuste del volumen descargado.

Para definir el polinomio que simula el tramo de perfil comprendido entre el punto de contacto y la pared se procede de forma análoga.

\section{RESULTADOS Y DISCUSIÓN}

Las figuras 3 y 4 permiten comparar los perfiles obtenidos con el modelo matemático y los resultados de un ensayo de carga realizado en un modelo a escala (1/10) del horno alto B de la compañía Aceralia para una carga estándar de coque y mineral, en la que se ha reproducido, a escala, el patrón de carga descrito en las tablas I y II. Los datos marcados en las tablas con la letra $m$ corresponden al modelo y los marcados con $r$ provienen del patrón real empleado por Aceralia para la carga del horno B.

Los perfiles del modelo físico se obtuvieron captando las imágenes de los perfiles tras la descarga del último anillo de coque y del último anillo de mineral en el modelo físico y procesándolas posteriormente para obtener el contorno de las capas de material.

\subsection{Capa de coque}

Las mayores discrepancias entre el cálculo y los resultados experimentales se dan para el caso de la

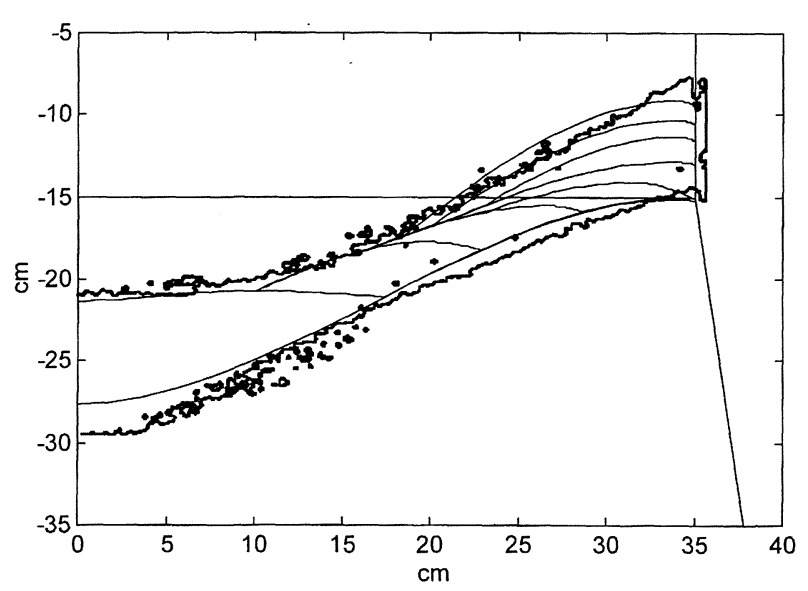

FIG. 3.- Comparación entre el perfil obtenido para la capa de coque en el modelo físico y el cálculo realizado en el modelo matemático. ( - modelo matemático, - - - modelo físico).

FIG. 3.- Comparison between the profile carried out from the physical model and the results obtained by the mathematical model for the coque layer. (- mathematical model, - - physical model).

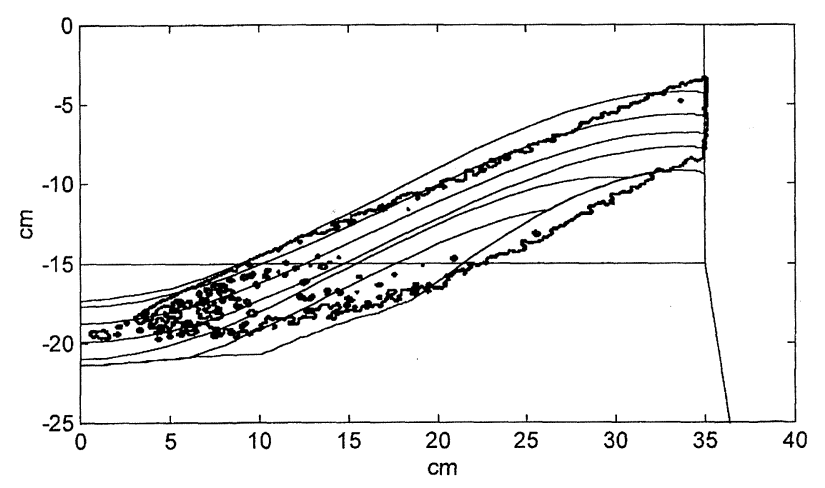

FIG. 4.- Comparación entre el perfil obtenido para la capa de mineral en el modelo físico y el cálculo realizado en el modelo matemático (- modelo matemático, - - - modelo físico).

FIG. 4.- Comparison between the profile carried out from the physical model and the results obtained by the mathematical model for the ore layer (- mathematical model, - - physical model).

capa de coque en las proximidades de la pared del horno para el material descargado en el último anillo. La razón de dicha discrepancia se encuentra en el efecto producido por la pared vertical del tragante del horno, que tiende a provocar una mayor acumulación de material cuando el punto de carga está próximo a la misma. Dicho efecto no se tuvo en cuenta a la hora de diseñar el modelo matemático, ya que, de hecho, el horno alto suele cargarse siempre por debajo del límite inferior del tragante, con lo que desaparece dicho efecto. La discrepancia en 
TABla I.- Distribución de la carga de coque por anillos para el horno y para el modelo. ( $r=$ horno, $m=$ modelo)

TABLE I.-Charging distribution pattern ( $r=$ blast furnace, $m=$ model $)$

\begin{tabular}{|c|l|c|c|c|c|c|c|c|c|c|c|c|}
\hline \multicolumn{10}{|c|}{ Coque } \\
\hline Anillo & 1 & 2 & 3 & 4 & 5 & 6 & 7 & 8 & 9 & 10 & 11 \\
\hline \multicolumn{1}{|c|}{ Ángulo $\left(^{\circ}\right)$} & 2 & 12 & 22 & 28 & 32 & 35 & 38 & 41 & 44 & 47 & 50 \\
\hline $\begin{array}{c}\text { Peso } \\
\text { descargado } \\
\text { (kg) }\end{array}$ & $\mathrm{r}$ & & 2714 & 2443 & 2036 & 1900 & 2307 & 2443 & 2443 & 2714 & & \\
\hline $\begin{array}{c}\text { Peso total } \\
\text { acumulado, }(\mathrm{kg})\end{array}$ & $\mathrm{r}$ & & 1,420 & 1,290 & 1,080 & 1,000 & 1,210 & 1,290 & 1,290 & 1,420 & & \\
\hline
\end{tabular}

TABLA II.- Distribución de la carga de coque por anillos para el horno y para el modelo. ( $r=$ horno, $m=$ modelo)

TABLE II.- Charging distribution pattern ( $r=$ blast furnace, $m=$ model)

\begin{tabular}{|c|c|c|c|c|c|c|c|c|c|c|c|c|}
\hline \multicolumn{13}{|c|}{ Mineral } \\
\hline Anillo & & 1 & 2 & 3 & 4 & 5 & 6 & 7 & 8 & 9 & 10 & 11 \\
\hline Ángulo (º) & & 2 & 12 & 22 & 28 & 32 & 35 & 38 & 41 & 44 & 47 & 50 \\
\hline $\begin{array}{c}\text { Peso } \\
\text { descargado } \\
(\mathrm{kg})\end{array}$ & $\begin{array}{l}\mathrm{r} \\
\mathrm{m}\end{array}$ & & & & $\begin{array}{l}12200 \\
6,321\end{array}$ & $\begin{array}{l}12200 \\
6,321\end{array}$ & $\begin{array}{c}12539 \\
6,536\end{array}$ & $\begin{array}{l}14064 \\
7,310\end{array}$ & $\begin{array}{c}15759 \\
8,170\end{array}$ & $\begin{array}{l}16098 \\
8,342\end{array}$ & & \\
\hline $\begin{array}{l}\text { Peso total } \\
\text { acumulado } \\
(\mathrm{kg})\end{array}$ & $\begin{array}{l}\mathrm{r} \\
\mathrm{m}\end{array}$ & & & & $\begin{array}{l}12200 \\
6,321\end{array}$ & $\begin{array}{l}24400 \\
12,642\end{array}$ & \begin{tabular}{|l|}
36939 \\
19,178
\end{tabular} & $\begin{array}{l}51003 \\
26,488\end{array}$ & $\begin{array}{c}66762 \\
34,658\end{array}$ & $\begin{array}{c}82860 \\
43,00 \\
0\end{array}$ & & \\
\hline $\begin{array}{c}\text { Peso } \\
\text { Pellets }(\mathrm{kg})\end{array}$ & $\begin{array}{l}\mathrm{r} \\
\mathrm{m}\end{array}$ & & & & $\begin{array}{l}7320 \\
3,793\end{array}$ & $\begin{array}{l}7320 \\
3,793\end{array}$ & $\begin{array}{l}7523 \\
3,921\end{array}$ & $\begin{array}{l}8438 \\
4,386\end{array}$ & $\begin{array}{l}9455 \\
4,902\end{array}$ & $\begin{array}{l}9659 \\
5,005\end{array}$ & & \\
\hline $\begin{array}{l}\text { Peso sinter } \\
\quad(\mathrm{kg})\end{array}$ & $\begin{array}{l}\mathrm{r} \\
\mathrm{m}\end{array}$ & & & & $\begin{array}{l}4880 \\
2,528\end{array}$ & $\begin{array}{l}4880 \\
2,528\end{array}$ & $\begin{array}{l}5016 \\
2,615\end{array}$ & $\begin{array}{l}5626 \\
2,924\end{array}$ & $\begin{array}{l}6304 \\
3,268\end{array}$ & $\begin{array}{l}6439 \\
3,337\end{array}$ & & \\
\hline
\end{tabular}

la zona central de la capa se explicará al analizar los resultados obtenidos para la capa de mineral. En líneas generales, el espesor medio obtenido por el modelo matemático difiere del resultado experimental en menos de $1 \mathrm{~cm}$, dejando aparte la discrepancia observada para la zona central de carga.

Es interesante hacer notar cómo el cálculo de la distribución carga por anillos permite obtener una capa convexa que se asemeja bastante a la distribución del modelo físico, con un mayor espesor en el centro del horno y en la pared y una zona intermedia más delgada. Este efecto se debe principalmente al patrón de carga empleado.

\subsection{Capa de mineral}

La figura 4 muestra la discrepancia existente entre el perfil de capa obtenido por el modelo mate- mático y el medido en el modelo físico. La carga de mineral sobre la capa anterior de coque provoca una fuerte pérdida de permeabilidad en la zona de descarga, lo que conlleva un incremento del flujo de gas en la zona central del horno, donde comienza a producirse una fluidización parcial de la capa de coque previamente cargada. Como consecuencia de ello, el coque tiende, en el centro del horno, a mezclarse con el mineral, lo que provoca la destrucción de la parte central de los anillos de mineral y la formación de una zona central en la que los materiales aparecen mezclados, efecto no modelado en este estudio.

Por tanto, la líneas que definen los perfiles correspondientes a los anillos de mineral llegan hasta el centro del horno. Sin embargo, las imágenes captadas del modelo físico, una vez procesadas, tienden a confundir la zona de mezcla con la de coque puro, debido a que el color de aquélla es más 
parecido al del coque que al del mineral. Este es el motivo de que, en la figura, la zona central de la capa de coque parece llegar más abajo que en el cálculo realizado por el modelo.

En la figura 5 se muestran, en un mismo gráfico, el perfil de la capa de coque, tal y como se observa en el modelo físico tras su carga, y el perfil de mineral cargado sobre él. La variación de la zona central de la interfase entre ambas capas ilustra el efecto anteriormente descrito.



FIG. 5.- Perfil de la capa de mineral sobre la capa previa de coque. La separación de la interfase en la zona central se debe a la fluidización de la capa de coque y posterior mezcla de materiales.

FIG. 5.- Ore layer profile onto a previous coque layer, showing the split of the boundary between both layers in the furnace central area due to fluidization of the coque layer and a subsequent mixture of materials.

\section{CONCLUSIONES}

El modelo desarrollado es capaz de estimar razonablemente la distribución de carga en el interior del horno alto.

El cálculo de los perfiles se realiza para cada anillo de carga, lo que dota al modelo de una gran flexibilidad, desligándolo de un patrón de carga determinado.

Las mayores discrepancias encontradas se deben a fenómenos dinámicos producidos por el paso de gas, tales como la destrucción de la zona central de las capas de mineral y coque y la mezcla de los materiales de las mismas.

Una versión del modelo desarrollado ha sido instalada en los ordenadores de proceso del horno alto $\mathrm{B}$ de la compañía Aceralia. El uso del modelo permite simular la distribución de carga en el horno, a la vez que se va realizando la carga del mismo.

\section{Agradecimiento}

Los autores agradecen a la Comunidad Europea del Carbón y del Acero la financiación económica del proyecto Advanced Modelization for Blast Furnace Control, en el cual se enmarca el presente trabajo, así como la concedida por la Comisión Interministerial de Ciencia y Tecnología (CICYT).

\section{REFERENCIAS}

(1) CHEN, J. ISIJ Int., 32, 1992: 1.259.

(2) ECSC Agreement 7210-AA/934. Draft Final Report. Raahe, Finland 1997.

(3) Blast furnace phenomena and modelling. Elsevier Applied Science. 1987: 364. 